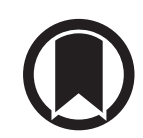

CrossMark

\title{
Clinical manifestations in primary ciliary dyskinesia: systematic review and meta-analysis
}

\author{
Myrofora Goutaki ${ }^{1,6}$, Anna Bettina Meier ${ }^{1,6}$, Florian S. Halbeisen ${ }^{1}$, \\ Jane S. Lucas ${ }^{2,3}$, Sharon D. Dell ${ }^{4}$, Elisabeth Maurer ${ }^{1}$, Carmen Casaulta ${ }^{5}$, \\ Maja Jurca ${ }^{1}$, Ben D. Spycher ${ }^{1}$ and Claudia E. Kuehni ${ }^{1}$
}

Affiliations: ${ }^{1}$ Institute of Social and Preventive Medicine, University of Bern, Bern, Switzerland. ${ }^{2}$ PCD Centre, NIHR Southampton Respiratory Biomedical Research Unit, University Hospital Southampton NHS Foundation Trust, Southampton, UK. ${ }^{3}$ Clinical and Experimental Sciences, Faculty of Medicine, University of Southampton, Southampton, UK. "Divisions of Respiratory Medicine and Child Health Evaluative Sciences, The Hospital for Sick Children, Toronto, ON, Canada. ${ }^{5}$ Dept of Pediatrics, University Children's Hospital of Bern, Bern, Switzerland. ${ }^{6}$ Both authors contributed equally.

Correspondence: Claudia E. Kuehni, Institute of Social and Preventive Medicine, University of Bern, Finkenhubelweg 11, 3012 Bern, Switzerland. E-mail: claudia.kuehnidispm.unibe.ch

ABSTRACT Few original studies have described the prevalence and severity of clinical symptoms of primary ciliary dyskinesia (PCD). This systematic review and meta-analysis aimed to identify all published studies on clinical manifestations of PCD patients, and to describe their prevalence and severity stratified by age and sex.

We searched PubMed, Embase and Scopus for studies describing clinical symptoms of $\geqslant 10$ patients with PCD. We performed meta-analyses and meta-regression to explain heterogeneity.

We included 52 studies describing a total of 1970 patients (range 10-168 per study). We found a prevalence of $5 \%$ for congenital heart disease. For the rest of reported characteristics, we found considerable heterogeneity ( $\mathrm{I}^{2}$ range $\left.68-93.8 \%\right)$ when calculating the weighted mean prevalence. Even after taking into account the explanatory factors, the largest part of the between-studies variance in symptom prevalence remained unexplained for all symptoms. Sensitivity analysis including only studies with testproven diagnosis showed similar results in prevalence and heterogeneity.

Large differences in study design, selection of study populations and definition of symptoms could explain the heterogeneity in symptom prevalence. To better characterise the disease, we need larger, multicentre, multidisciplinary, prospective studies that include all age groups, use uniform diagnostics and report on all symptoms.

@ERSpublications

Review of the clinical manifestations of PCD found between-study variation; large prospective studies needed http://ow.ly/Y5GC300Sw73

This article has supplementary material available from erj.ersjournals.com

Received: Nov 192015 | Accepted after revision: May 242016 | First published online: Aug 042016

Support statement: Primary ciliary dyskinesia (PCD) research at the Institute of Social and Preventive Medicine Bern and University Hospital Southampton receives funding from the European Union's Seventh Framework Programme under EG-GA No. 35404 BESTCILIA (Better Experimental Screening and Treatment for Primary Ciliary Dyskinesia). The researchers participate in the network of COST Action BEAT-PCD (Better Evidence to Advance Therapeutic Options for PCD) (BM 1407). M. Goutaki, J.S. Lucas and C.E. Kuehni are members of the European Respiratory Society PCD taskforce for PCD diagnostics (ERS TF-2014-04). B.D. Spycher is supported by a Swiss National Science Foundation fellowship (PZ00P3_147987). M. Jurca is supported by a Swiss National Science Foundation grant (PDFMP3 137033). Sharon D. Dell is funded by grant support U54HL096458 from the National Institutes of Health (NIH) through the Genetic Disorders of Mucociliary Clearance Consortium, an initiative of the NIH Office of Rare Diseases Research at the National Center for Advancing Translational Science and the National Heart, Lung and Blood Institute. Funding information for this article has been deposited with the Open Funder Registry.

Conflict of interest: None declared.

Copyright CERS 2016 


\section{Introduction}

Primary ciliary dyskinesia (PCD) is a rare inherited disease which affects ciliary structure and function. As with most orphan diseases, PCD research has focused on pathology and diagnostics. PCD leads to severely impaired mucociliary clearance and a wide variety of symptoms primarily affecting the respiratory system [1]. Productive cough, rhinitis and recurrent infections of the upper and lower respiratory tract have been described as leading symptoms [2, 3]. Manifestations from other systems have also been reported and about half the patients have been described to present with situs inversus [4]. In addition, many males with PCD have immobile spermatozoa or dysfunction of cilia in the epididymal duct, leading to infertility [5]. The prevalence of the disease is estimated to be between 1:2000 and 1:40000 [3], but it is underdiagnosed [6].

Information about clinical symptoms of PCD is derived mainly from case series and nonsystematic reviews reflecting expert opinion. There are few original studies; most include a small study population, consisting primarily of paediatric patients. Original publications describing the full spectrum of symptoms are scarce and there are few data on less common symptoms. In many diseases it is known that symptoms evolve and change with age, but few studies describe PCD patients from different age groups and show how symptoms change over time. PCD patients comprise a relatively heterogeneous group, as diagnostics and management approaches vary between centres $[1,7]$. PCD diagnosis is still not uniform internationally and most recommended tests are not available in many centres and countries, so clinical manifestations continue to play an important role in the diagnosis of PCD.

In this systematic review and meta-analysis, we aimed to identify all published studies presenting clinical symptoms and signs in PCD patients and describe the reported prevalence of all clinical manifestations. This includes the prevalence of upper and lower respiratory symptoms as well as less common clinical findings. In addition, we aimed to describe differences in prevalence and severity of findings in different age groups.

\section{Methods}

We developed a protocol for the systematic review beforehand, which is described in the following sections.

\section{Search strategy}

We searched the online databases PubMed, Embase and Scopus to identify studies describing clinical manifestations in patients with PCD. In order to build a search term that would identify as many studies as possible, we first performed a pilot search. We searched for studies that were published between January 1980 and April 2015 including published abstracts. We conducted this search without any restrictions in language or study design.

The search was performed using the following terms:

PubMed: ((("kartagener syndrome"[tiab] OR "primary ciliary dyskinesia”[tiab] OR "ciliary motility disorder" [tiab] OR "immotile cilia syndrome"[tiab]) OR “ciliary motility disorders"[mh]) AND ("clinical symptoms"[All Fields] OR "clinical manifestations"[All Fields] OR "clinical presentation"[All Fields])) OR ((("kartagener syndrome"[tiab] OR "primary ciliary dyskinesia”[tiab] OR "ciliary motility disorder"[tiab] OR “immotile cilia syndrome"[tiab]) OR "ciliary motility disorders"[mh]) AND (patients[tiab] OR subjects[tiab] OR participants[tiab] OR “cases”[tiab])) AND (“1980/01/01”[PDAT] : “2015/04/30”[PDAT])

Embase: "primary ciliary dyskinesia"/syn OR "primary ciliary dyskinesia" OR "kartagener syndrome"/syn OR "kartagener syndrome” AND ("clinical symptoms":ab OR "clinical manifestations":ab OR "clinical presentation”:ab OR patients:ab OR subjects:ab OR cases:ab OR participants:ab) AND ([article]/lim OR [article in press]/lim OR [conference abstract]/lim OR [conference paper]/lim) AND [1980-2015]/py

Scopus: (((TITLE-ABS-KEY(primary ciliary dyskinesia) AND PUBYEAR > 1979) OR (TITLE-ABS-KEY (immotile cilia syndrome) AND PUBYEAR > 1979) OR (TITLE-ABS-KEY(kartagener syndrome) AND PUBYEAR > 1979)) AND (TITLE-ABS-KEY(“clinical symptoms" OR "clinical manifestations" OR "clinical presentation”) AND PUBYEAR > 1979)) OR (((TITLE-ABS-KEY(primary ciliary dyskinesia) AND PUBYEAR > 1979) OR (TITLE-ABS-KEY(immotile cilia syndrome) AND PUBYEAR > 1979) OR (TITLE-ABS-KEY(kartagener syndrome) AND PUBYEAR > 1979)) AND (TITLE-ABS-KEY(patients OR subjects OR participants OR cases) AND PUBYEAR > 1979))

After identifying all eligible studies, we checked for additional citations in their reference lists. We used the Endnote X5 (Thomson Reuters, Philadelphia, PA, USA) citation manager.

\section{Definition of PCD patients}

We defined PCD patients as all patients reported by the authors as being diagnosed with PCD. This included a wide range of inclusion criteria, ranging from patients with a clinical diagnosis to those with 
positive results from the different available diagnostic tests (electron microscopy, light or high-speed video microscopy, nasal nitric oxide (nNO) and genetics).

\section{Study selection}

We included studies containing information on clinical manifestations of patients with PCD with a study population of $\geqslant 10$ individuals. We excluded publications based on the following exclusion criteria: not original studies, studies that were not topic related or did not contain any clinical information (e.g. describing diagnostics or genetics) and studies describing other rare ciliary syndromes such as Joubert or Meckel-Gruber syndrome.

We decided upon inclusion initially by screening the titles and abstracts. From our pilot search we realised that many studies containing information on clinical manifestations did not explicitly articulate this in the title or abstract. For this reason, we decided to screen the full text of all studies which described an original study population of PCD patients and thus had a high probability of containing clinical information in the full text, even if it was not mentioned in the title or abstract. After reading the full text of all potentially eligible studies, the final decision on whether to include them in the review or not was made by two reviewers. During the final step of inclusion, we excluded studies that did not contain any clinical information. The two reviewers decided independently, and in case of disagreement, a consensus decision was reached after discussion.

\section{Overlapping study population}

We identified all studies which might have described the same study population, in order to avoid including the same patients multiple times in our review. We compared the author list, country of origin and department where the study took place and when we noticed a considerable overlap in the study population, we always included in the quantitative synthesis the study that was published most recently and included information on a larger number of patients and/or more clinical manifestations. When the studies were published $\geqslant 10$ years apart, we included them both, as we believe there was little chance of significant overlap. Where the possibility of an overlap was not clear, we contacted the investigators to clarify it.

\section{Data extraction}

Using the software Epidata 3.1 (www.epidata.dk), we extracted the following information from all studies, including the ones with overlapping populations: author- and publication-specific information, study characteristics and information on reported clinical manifestations of PCD patients. Specifically, we extracted publication details (e.g. author names, journal and year of publication and country and centre of corresponding author) and study characteristics (e.g. years of study performance, study design, inclusion and exclusion criteria, study population size, country where the study took place, type of clinic, age of participants and age stratification of clinical manifestations). Secondly, we extracted extensive information on all reported clinical manifestations of PCD patients, such as situs inversus, lower and upper respiratory symptoms, neonatal symptoms and other nonrespiratory findings (e.g. congenital heart disease or infertility). We extracted the number of affected individuals in each study and calculated the prevalence of the reported clinical manifestations. Where only percentages of affected patients were given, we calculated the number of patients affected and then the prevalence.

\section{Meta-analysis}

We used a random effects model for binomial data to perform meta-analyses on the transformed prevalence (Freeman-Tukey double arcsine transformation) of clinical manifestations [8-10] and to assess the heterogeneity $\left(\mathrm{I}^{2}\right)$ between studies [11]. Diagnosis of PCD has evolved significantly over time and could have influenced the characteristics of the patients included in the eligible studies. Therefore, we performed subgroup meta-analyses, which excluded studies where PCD diagnosis was based only on clinical manifestations or where information on diagnostics was not available.

To investigate reasons for heterogeneity we then fitted meta-regression models considering the following explanatory factors one at a time: type of clinic (general paediatrics, paediatric pulmonology, adult pulmonology, ear-nose-throat (ENT) clinic or other), age of patients (adults, children or both), publication year (published before 1994, 1995-2004 and since 2005), number of patients included $(<20,21-50,51-100$ and $>100$ patients), study design (retrospective or prospective) and level of diagnostic certainty (clinical diagnosis, diagnosis proven by electron microscopy or diagnosis proven by combination of electron microscopy and other tests (video microscopy, nNO or genetics)). Studies in which the inclusion criteria influenced the prevalence of some clinical manifestations were excluded from the meta-analyses and meta-regression for these characteristics. For instance, studies describing patients with Kartagener syndrome were excluded from the meta-analyses on prevalence of situs anomalies, bronchiectasis and sinusitis. We also excluded these studies from the meta-analysis on prevalence of congenital heart disease, as patients with 
situs anomalies have a higher probability of having congenital heart disease than patients with situs solitus. Statistical analysis was performed using $\mathrm{R}$ software version 3.2 (www.r-project.org) using the meta package (version 4.2) and specifically the commands metaprop and metareg.

\section{Results}

Search

After excluding duplicates identified by more than one database (Pubmed, Embase or Scopus) our search identified 1210 articles (figure 1). First, we screened for inclusion and exclusion criteria by reading through titles and abstracts and excluded 1109 articles. It was not possible to find the abstract or the full text for 16 studies. This resulted in 101 articles. After reading the full texts, we excluded another 49 articles; 19 did not contain any clinical information and 30 had a largely overlapping study population [4, 12-40].

Three pairs of studies had partially overlapping study populations, but unique data, hence were all included in the quantitative synthesis. Studies by Pedersen and Stafanger [41] and Mygind and Pedersen [42] described different symptoms in the same population. Studies by McManus et al. [43] and JAIN et al. [44] had only a small partial overlap and provided mostly unique information. Articles by SHAPIRO et al. [45] and DAvis et al. [46] had a partial overlap in study population, but one described only situs anomalies and the other more clinical characteristics. Ultimately, we included a total of 52 studies.

\section{Study characteristics}

Table 1 lists the included studies and describes their characteristics. The 51 articles $[2,41-90]$ and one conference abstract [91] included described a total of 1970 patients, with a mean number of 38 patients per study (range 10-168). Nearly half of the studies originated from paediatric clinics or paediatric pulmonology departments, 11 came from ENT departments, four from adult pulmonology departments and 12 from other departments, such as diagnostic laboratories, radiology and pathology departments (table 2). Two-thirds were single-centre studies. More than half of the studies (56\%) were published in the past 10 years (since 2005). Studies were relatively small, with most including $<50$ patients. Most studies $(\mathrm{n}=31)$ came from Europe, 10 from Asia, eight from North America, two from South America and one from Australia. 17 studies included only children (age <18 years), three included only adults (age $\geqslant 18$ years) and 32 studies described a study population of mixed age, consisting mainly of children with only few adults. Among those 32 studies, only 11 described the clinical data stratified by age group. Symptoms were assessed retrospectively in most studies $(n=37,71 \%)$. PCD diagnosis was established in

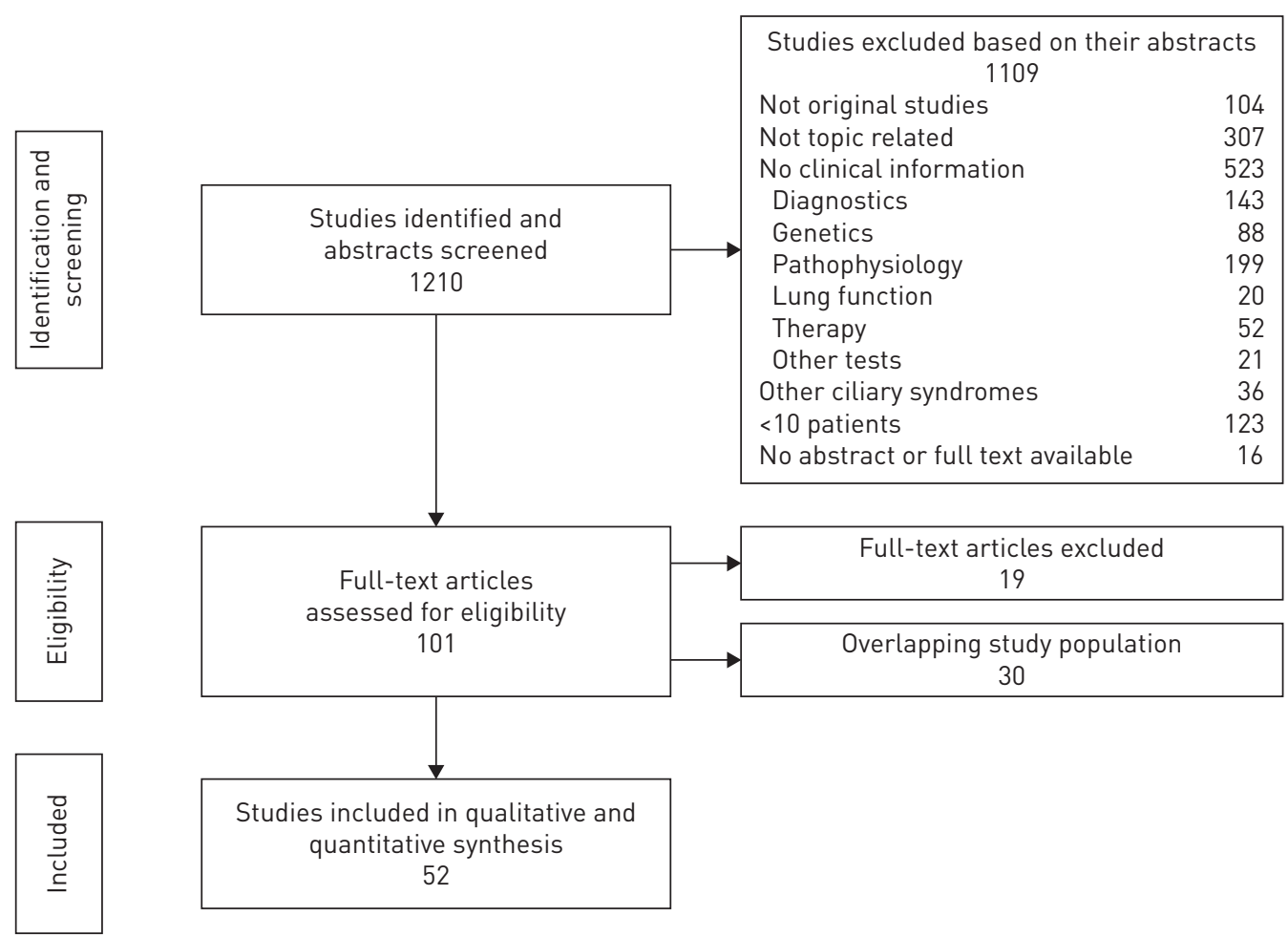

FIGURE 1 Flow chart describing the selection procedure. Data are presented as $\mathrm{n}$. 
TABLE 1 Detailed characteristics of included studies describing clinical manifestations of primary ciliary dyskinesia (PCD), stratified by age group of participants

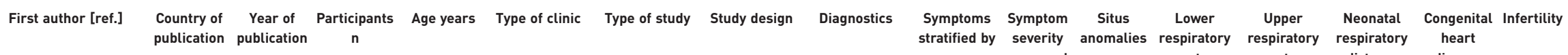
$\begin{array}{ccc}\text { publication publication } n & \text { stratified by } & \text { severity } \\ \text { assessed }\end{array}$ symptoms symptoms distress

\begin{tabular}{|c|c|c|c|c|c|c|c|c|c|c|c|c|c|c|c|c|}
\hline \multicolumn{17}{|l|}{ Children } \\
\hline ALSAADI [48] & Saudi Arabia & 2013 & 22 & $11^{\pi}$ & Paediatric & Case-control & $\mathrm{R}$ & $E M+n N O$ & - & - & + & + & + & - & - & - \\
\hline BARLOCCO [52] & Italy & 1991 & 28 & $8^{\pi}(0-18)$ & $\begin{array}{l}\text { Paediatric } \\
\text { pulmonology }\end{array}$ & Case series & $\mathrm{P}$ & EM & - & - & + & + & + & - & - & - \\
\hline BEUCHER [53] & France & 2011 & 17 & $7^{\pi}$ & $\begin{array}{l}\text { Paediatric } \\
\text { pulmonology }\end{array}$ & Case series & $\mathrm{R}$ & $E M+n N O$ & - & - & + & - & + & + & - & - \\
\hline BusQuets [55] & Spain & 2013 & 35 & & $\begin{array}{l}\text { Paediatric } \\
\text { pulmonology }\end{array}$ & Case series & $\mathrm{R}$ & EM & - & - & - & + & - & - & - & - \\
\hline Davis [46] & USA & 2015 & 118 & $8^{7}(5-11)$ & Combination & Case series & $\mathrm{P}$ & $\begin{array}{c}\mathrm{EM}+\mathrm{nNO}+ \\
\text { genetics }\end{array}$ & - & - & + & + & + & + & - & - \\
\hline ENDERBY [91] ${ }^{\#}$ & UK & 2010 & 17 & $9^{+}(4-17)$ & Paediatrics & Case series & $R$ & $\begin{array}{l}\text { Clinical diagnosis } \\
\text { only }\end{array}$ & - & - & + & + & + & + & - & - \\
\hline HosIE [90] & Australia & 2015 & 84 & $6^{+}(0-18)$ & Paediatrics & Cohort & $\mathrm{R}$ & $E M+V M+n N O$ & - & - & + & + & + & + & + & - \\
\hline JAIN [44] & UK & 2007 & 89 & & $\begin{array}{l}\text { Paediatric } \\
\text { pulmonology }\end{array}$ & Case series & $\mathrm{R}$ & $E M+V M+n N O$ & - & - & + & + & + & + & - & - \\
\hline KoRPPI [65] & Finland & 2011 & 12 & & Paediatrics & Cohort & $\mathrm{R}$ & EM & - & - & + & + & - & - & - & - \\
\hline Min [68] & South Korea & 1995 & 19 & $10^{\pi 1}(5-15)$ & Paediatrics & Case series & $\mathrm{R}$ & EM & - & - & + & + & + & - & - & - \\
\hline MuLLoWney [87] & Canada & 2014 & 55 & $11^{\pi}$ & $\begin{array}{l}\text { Paediatric } \\
\text { pulmonology }\end{array}$ & Case-control & $\mathrm{R}$ & $\begin{array}{c}\mathrm{EM}+\mathrm{nNO}+ \\
\text { genetics }\end{array}$ & - & - & + & + & - & + & + & - \\
\hline O'Callaghan [70] & UK & 2010 & 19 & & $\begin{array}{l}\text { PCD diagnostic } \\
\text { centre }\end{array}$ & Case series & $\mathrm{R}$ & $E M+V M$ & - & - & + & + & - & + & - & - \\
\hline RACHINSKII [75] & Russia & 1993 & 24 & & Paediatrics & Case series & $\mathrm{R}$ & EM & - & - & + & + & + & - & + & - \\
\hline RUtISHAUSER [76] & Switzerland & 2000 & 30 & & Paediatrics & Case series & $\mathrm{R}$ & $E M+V M$ & - & - & + & + & + & - & + & - \\
\hline Vallet [81] & France & 2013 & 60 & $0-15$ & $\begin{array}{l}\text { Paediatric } \\
\text { pulmonology }\end{array}$ & Case series & $\mathrm{R}$ & $E M+V M$ & - & - & + & + & + & + & - & - \\
\hline WoLter [84] & Canada & 2012 & 31 & $7^{71}(0-17)$ & ENT & Case series & $\mathrm{R}$ & $\mathrm{EM}+\mathrm{nNO}$ & - & - & + & + & + & + & - & - \\
\hline $\mathrm{Xu}[85]$ & China & 2008 & 26 & & Paediatrics & Case series & $\mathrm{R}$ & EM & - & - & + & + & + & - & - & - \\
\hline \multicolumn{17}{|l|}{ Adults } \\
\hline AfzeLIus [47] & Sweden & 1983 & 29 & & $\begin{array}{l}\text { PCD diagnostic } \\
\text { centre }\end{array}$ & Case series & $\mathrm{R}$ & EM & - & - & + & - & - & - & - & + \\
\hline CAMner [56] & Sweden & 1983 & 20 & $30^{\pi}(19-40)$ & $\begin{array}{l}\text { Adult } \\
\text { pulmonology }\end{array}$ & Case series & $\mathrm{R}$ & $\begin{array}{l}\text { Clinical diagnosis } \\
\text { only }\end{array}$ & - & - & + & + & + & - & - & - \\
\hline SмIт [77] & Canada & 1996 & 21 & $46^{\pi}(24-66)$ & $\begin{array}{l}\text { Adult } \\
\text { pulmonology }\end{array}$ & Case series & $\mathrm{R}$ & $E M+V M$ & - & - & + & + & - & - & - & - \\
\hline \multicolumn{17}{|l|}{ Children and adults } \\
\hline ARMENGOT [49] & Spain & 2012 & 25 & $28^{n}(1-66)$ & ENT & Case series & $\mathrm{P}$ & $E M+V M$ & + & - & + & + & + & - & - & - \\
\hline ARMENGOT [50] & Spain & 1995 & 14 & $24^{\Uparrow}(5-50)$ & ENT & Case series & $\mathrm{P}$ & EM & - & - & + & + & + & - & - & - \\
\hline BAI [51] & China & 2014 & 10 & $35^{\Uparrow}(6-56)$ & ENT & Case series & $\mathrm{R}$ & $\begin{array}{c}\mathrm{EM}+\mathrm{nNO}+ \\
\text { genetics }\end{array}$ & + & - & + & + & + & - & - & + \\
\hline Boon [39] & Belgium & 2014 & 168 & $18^{+}$ & $\begin{array}{l}\text { PCD diagnostic } \\
\text { centre }\end{array}$ & Cohort & $\mathrm{R}$ & $\begin{array}{c}\mathrm{EM}+\mathrm{VM}+\mathrm{nNO}+ \\
\text { genetics }\end{array}$ & - & - & + & + & + & + & + & - \\
\hline Braun [54] & France & 2005 & 35 & & ENT & Case series & $\mathrm{R}$ & $E M+V M$ & - & - & + & + & - & + & + & - \\
\hline CHIN [89] & China & 2002 & 73 & $0-48$ & Pathology & Case series & $\mathrm{R}$ & $E M+n N O$ & - & - & - & + & + & - & - & - \\
\hline \multirow[t]{2}{*}{ DE Boode [57] } & The & 1989 & 34 & $23^{n}(6-55)$ & Paediatric & Case series & $\mathrm{R}$ & $E M+V M$ & - & - & + & + & + & - & - & - \\
\hline & Netherlands & & & & & & & & & & & & & & & \\
\hline EL-SAYED [58] & Saudi Arabia & 1997 & 16 & $18^{11}(2-46)$ & ENT & Case series & $\mathrm{P}$ & EM & + & - & + & + & + & - & - & - \\
\hline GoYAL [59]"\# & India & 1987 & 11 & & $\begin{array}{c}\text { Adult } \\
\text { pulmonology }\end{array}$ & Case series & $R$ & $\begin{array}{l}\text { Clinical diagnosis } \\
\text { only }\end{array}$ & + & - & + & + & + & - & - & - \\
\hline GREENSTONE [60] & UK & 1988 & 30 & $19^{\pi 1}(0-51)$ & Cardiothoracic & Case series & $\mathrm{P}$ & $E M+V M$ & _- & _- & + & + & + & - & _ & + \\
\hline HeLLinckx [61] & Belgium & 1998 & 12 & $1-32$ & $\begin{array}{l}\text { Paediatric } \\
\text { pulmonology }\end{array}$ & Case series & $\mathrm{R}$ & $E M+V M$ & - & - & - & + & - & - & - & - \\
\hline HoLzMANn [62] & Switzerland & 2000 & 10 & & ENT & Case series & $\mathrm{R}$ & $E M+V M$ & - & - & + & + & + & + & - & - \\
\hline
\end{tabular}


TABLE 1 Continued

First author [ref.] Country of Year of Participants Age years Type of clinic Type of study Study design Diagnostics Symptoms Symptom Situs Lower Upper Neonatal Congenital Infertility publication publication $n \quad$ stratified by severity anomalies respiratory respiratory respiratory heart

\begin{tabular}{|c|c|c|c|c|c|c|c|c|c|c|c|c|c|c|c|c|}
\hline & & & & & & & & & & & & & & & & \\
\hline INIGGEZZ [63] & Chile & 2007 & 33 & & ENT & Case series & $\mathrm{R}$ & EM & - & - & + & + & + & - & - & - \\
\hline KaWAKAMI [64] & Japan & 1996 & 48 & $38^{\text {ๆ }}(17-72)$ & $\begin{array}{l}\text { Paediatric } \\
\text { pulmonology }\end{array}$ & Case series & $R$ & EM & - & - & + & + & + & - & - & - \\
\hline LESIC [66] & Austria & 2009 & 13 & & Paediatrics & Case series & $\mathrm{R}$ & EM & - & - & + & + & + & - & - & + \\
\hline MARTHIN [67] & Denmark & 2010 & 74 & $19^{+}(6-70)$ & $\begin{array}{l}\text { PCD reference } \\
\text { centre }\end{array}$ & Case series & $\mathrm{R}$ & $E M+V M+n N O$ & - & - & + & + & + & + & + & - \\
\hline McManus [43] $]^{\#}$ & UK & 2003 & 93 & $23^{n}(4-66)$ & Psychology & Case series & $P$ & & - & - & + & + & + & - & - & - \\
\hline Montella [69] & Italy & 2009 & 13 & $15^{+}(10-29)$ & Paediatrics & Case series & $\mathrm{P}$ & $E M+V M$ & + & - & + & + & - & + & - & - \\
\hline MYGIND [42] ${ }^{\#}$ & Denmark & 1983 & 27 & $24^{\pi}(4-56)$ & ENT & Case series & $\mathrm{P}$ & $\begin{array}{c}\text { Clinical diagnosis } \\
\text { only }\end{array}$ & + & - & + & + & + & - & - & - \\
\hline Noone [2] & USA & 2004 & 78 & $27^{\natural}(1-73)$ & Combination & Case series & $\mathrm{P}$ & $\mathrm{EM}+\mathrm{VM}+\mathrm{nNO}$ & + & - & + & + & + & + & - & - \\
\hline Октем [71] & Turkey & 2013 & 29 & $10^{1}(0-24)$ & Paediatrics & Case-control & $\mathrm{P}$ & EM & - & - & - & + & + & - & - & - \\
\hline Oเм [72] & Brazil & 2011 & 12 & $12^{\pi}(1-19)$ & $\begin{array}{l}\text { Paediatric } \\
\text { pulmonology }\end{array}$ & Case series & $R$ & EM & + & - & + & + & - & - & + & - \\
\hline Pedersen [41] & Denmark & 1983 & 27 & & Paediatrics & Case series & $\mathrm{P}$ & $E M+V M$ & + & - & + & + & + & & + & + \\
\hline PIFFERI [73] & Italy & 2010 & 78 & $21^{\pi}(2-49)$ & Paediatrics & Case series & $\mathrm{P}$ & $E M+V M$ & - & - & + & + & + & - & + & - \\
\hline PLesec [74] & USA & 2008 & 13 & $15^{\pi}(1-49)$ & Pathology & Case series & $\mathrm{R}$ & EM & + & - & + & + & + & - & + & + \\
\hline SHAPIRO [45] & USA & 2014 & 35 & $18^{n}(2-58)$ & Combination & Case series & $\mathrm{R}$ & $\begin{array}{c}\mathrm{EM}+\mathrm{nNO}+ \\
\text { genetics }\end{array}$ & - & - & + & + & + & + & + & - \\
\hline SOMMER [78] & Germany & 2011 & 44 & $29 \pi$ & ENT & Case series & $\mathrm{R}$ & & - & - & + & - & + & - & - & - \\
\hline Sturgess [79] & Canada & 1986 & 46 & & Paediatrics & Case series & $\mathrm{R}$ & EM + genetics & + & - & + & + & + & - & - & - \\
\hline Tolusakow [80]" & Russia & 1981 & 23 & $3-43$ & $\begin{array}{c}\text { Adult } \\
\text { pulmonology }\end{array}$ & Case series & P & $\begin{array}{c}\text { Clinical diagnosis } \\
\text { only }\end{array}$ & - & - & + & + & + & - & - & - \\
\hline VAN DER BAAN [82] & $\begin{array}{c}\text { The } \\
\text { Netherlands }\end{array}$ & 1991 & 36 & $25^{\Uparrow}(1-59)$ & ENT & Case series & P & $E M+V M$ & - & - & + & - & - & - & - & - \\
\hline WANG [83] & China & 2009 & 24 & $29^{11}(4-63)$ & Radiology & Case series & $\mathrm{R}$ & EM & - & - & + & + & + & - & + & + \\
\hline YIALLOUROS [86] & Cyprus & 2015 & 30 & $24^{n}(1-64)$ & Paediatrics & Cohort & $\mathrm{R}$ & $E M+V M+n N O$ & - & - & + & + & + & + & - & - \\
\hline
\end{tabular}

Data are presented as mean (range) or median (range) or range, unless otherwise stated. R: retrospective; EM: electron microscopy; nNO: nasal nitric oxide; P: prospective; VM: light or high-frequency video microscopy; ENT: ear, nose and throat. \#: studies excluded from subgroup meta-analyses; ${ }^{\text {ๆ: }}$ mean; ${ }^{+}$: median. 


\begin{tabular}{|c|c|}
\hline Total studies & $52(100)$ \\
\hline \multicolumn{2}{|l|}{ Type of clinic } \\
\hline Paediatric department & $16(33)$ \\
\hline Paediatric pulmonology department & $9(17)$ \\
\hline Adult pulmonology department & $4(8)$ \\
\hline ENT department & $11(21)$ \\
\hline Other department & $12(21)$ \\
\hline \multicolumn{2}{|l|}{ Number of centres } \\
\hline Single centre & $35(66)$ \\
\hline Multicentre & 17 (33) \\
\hline \multicolumn{2}{|l|}{ Publication period } \\
\hline Prior to 1994 & $12(23)$ \\
\hline 1995-2004 & $11(21)$ \\
\hline Since 2005 & $29(56)$ \\
\hline \multicolumn{2}{|l|}{ Study size (number of patients) } \\
\hline$\leqslant 20$ & $15(29)$ \\
\hline $21-50$ & $25(48)$ \\
\hline $50-100$ & $9(14)$ \\
\hline$>100$ & $3(6)$ \\
\hline \multicolumn{2}{|l|}{ Study region } \\
\hline Europe & $31(60)$ \\
\hline Asia & $10(19)$ \\
\hline North America & $8(15)$ \\
\hline South America & $2(4)$ \\
\hline Australia & $1(2)$ \\
\hline \multicolumn{2}{|l|}{ Age of participants } \\
\hline Children $<18$ years & $17(33)$ \\
\hline Adults $\geqslant 18$ years & $3(6)$ \\
\hline Children and adults & $32(62)$ \\
\hline \multicolumn{2}{|l|}{ Study design } \\
\hline Retrospective & $37(71)$ \\
\hline Prospective & $15(29)$ \\
\hline \multicolumn{2}{|l|}{ Diagnostics performed } \\
\hline Clinical diagnosis only & $5(10)$ \\
\hline Diagnosis proven using EM & $16(31)$ \\
\hline Diagnosis proven using EM plus other tests" & $29(56)$ \\
\hline No information on diagnostics available & $2(4)$ \\
\hline \multicolumn{2}{|l|}{ Health conditions } \\
\hline Situs anomalies & $48(92)$ \\
\hline Lower respiratory tract conditions & $48(92)$ \\
\hline Cough & $29(56)$ \\
\hline Sputum production & $24(46)$ \\
\hline Lower respiratory tract infections & $27(52)$ \\
\hline Bronchiectasis & $34(65)$ \\
\hline Upper respiratory tract conditions & $41(79)$ \\
\hline Rhinitis & $28(54)$ \\
\hline Otitis media & $26(50)$ \\
\hline Sinusitis & $32(62)$ \\
\hline Hearing impairment & $14(27)$ \\
\hline Grommets & $12(23)$ \\
\hline Nasal polyps & $14(27)$ \\
\hline \multicolumn{2}{|l|}{ Other conditions } \\
\hline Neonatal respiratory distress & 17 (33) \\
\hline Congenital heart disease & $13(25)$ \\
\hline Infertility & $7(14)$ \\
\hline Hydrocephalus & $3(6)$ \\
\hline Retinitis pigmentosa & $2(4)$ \\
\hline Renal symptoms & $0(0)$ \\
\hline Information for different age groups & $11^{11}(34)$ \\
\hline Information on severity & $0(0)$ \\
\hline
\end{tabular}

Data are presented as $\mathrm{n}(\%)$. ENT: ear, nose and throat; EM: electron microscopy. ${ }^{\#}$ : one or more of the following: nasal nitric oxide, high-frequency videomicroscopy or light microscopy or genetics; " studies including children and adults. 
different ways: 16 studies assessed ciliary ultrastructure only using electron microscopy and 29 with additional diagnostic tests (one or more of the following: nNO, video microscopy and genetics). Five studies diagnosed patients only by clinical presentation; two did not describe the diagnostic evaluation of the patients. Most studies described situs anomalies (92\%) and lower (92\%) and upper (79\%) respiratory symptoms. Other manifestations and health problems were seldom reported: 17 (33\%) studies reported on neonatal respiratory distress, $13(25 \%)$ on congenital heart disease, seven (14\%) on infertility, three $(6 \%)$ on hydrocephalus, two (4\%) on retinitis pigmentosa and none on renal symptoms.

\section{Prevalence of clinical manifestations}

Online supplementary table S1 describes the prevalence of commonly reported clinical manifestations in the included studies, categorised by country of origin, including studies with overlapping populations. For all reported characteristics the prevalence varied widely between studies and our analysis showed considerable heterogeneity ( $\mathrm{I}^{2}$ range $68-94 \%$ ). Figures $2-4$ and online supplementary figures S1-S11 describe the prevalence of symptoms in the different studies. In the following text and figures we report all symptoms described in five or more studies.

\section{Situs anomalies}

$41(79 \%)$ studies explicitly reported situs inversus and seven (14\%) reported only the cardiac situs of the patients or used the term situs ambiguus without any further specification. To calculate the prevalence, we summed these symptoms up under the designation of situs anomalies. After excluding studies that focused on describing patients with Kartagener syndrome, which had a high prevalence of situs anomalies (up to $100 \%$ ), the prevalence of situs anomalies in the 43 eligible studies ranged from $11 \%$ to $90 \%$ (weighted mean $49 \%$ with a heterogeneity of $\mathrm{I}^{2}=71 \%$; online supplementary figure $\mathrm{S} 1$ ).

\section{Lower respiratory symptoms}

Cough was reported in 29 (55.8\%) studies and prevalence varied from 14\% to $100 \%$ with a weighted mean of $88 \%$ (online supplementary figure S2). Sputum production was reported for $15-100 \%$ of patients (weighted mean $89 \%$ ) in the 24 (46\%) studies where it was described (figure 2). Lower respiratory infections, including

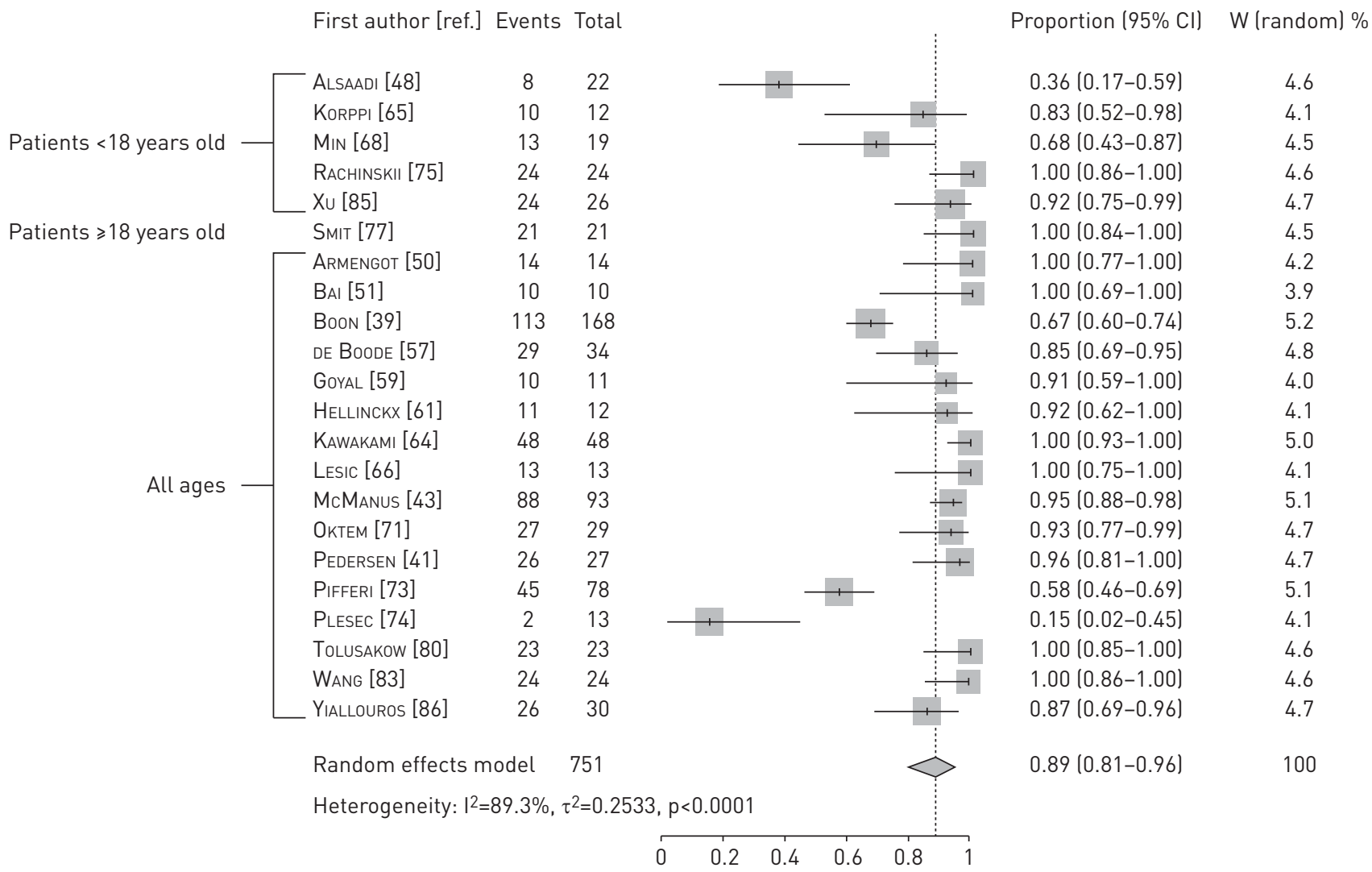

FIGURE 2 Sputum production in primary ciliary dyskinesia patients: forest plot showing the heterogeneity and weighted mean prevalence in the included publications. 
First author [ref.] Events Total

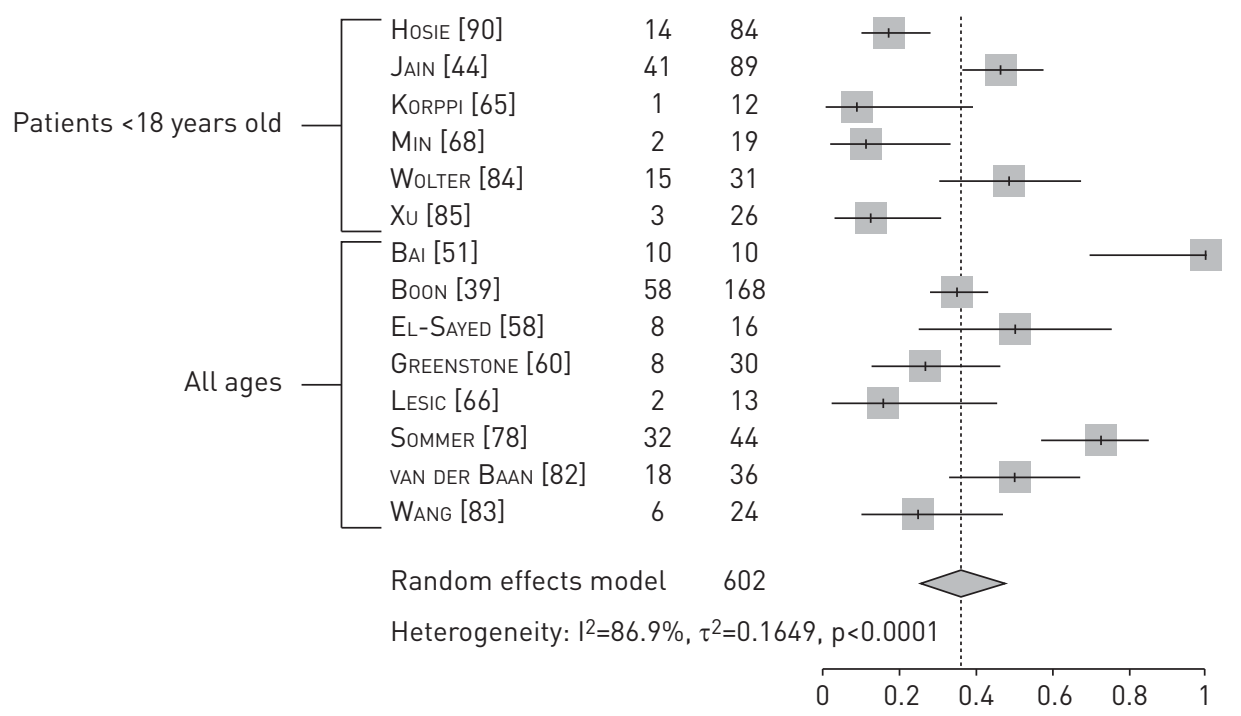

Proportion (95\% Cl) W (random) \%

$\begin{array}{ll}0.17(0.09-0.26) & 8.2 \\ 0.46(0.35-0.57) & 8.3 \\ 0.08(0.00-0.38) & 5.9 \\ 0.11(0.01-0.33) & 6.7 \\ 0.48(0.30-0.67) & 7.4 \\ 0.12(0.02-0.30) & 7.2 \\ 1.00(0.69-1.00) & 5.6 \\ 0.35(0.27-0.42) & 8.5 \\ 0.50(0.25-0.75) & 6.4 \\ 0.27(0.12-0.46) & 7.3 \\ 0.15(0.02-0.45) & 6.1 \\ 0.73(0.57-0.85) & 7.8 \\ 0.50(0.33-0.67) & 7.6 \\ 0.25(0.10-0.47) & 7.1 \\ 0.36(0.25-0.48) & 100\end{array}$

FIGURE 3 Hearing impairment in primary ciliary dyskinesia patients: forest plot showing the heterogeneity and weighted mean prevalence in the included publications.

pneumonia, were also common, reported in 27 (52\%) studies with a weighted mean prevalence of $72 \%$ (range 15-100\%; online supplementary figure S3). Prevalence of bronchiectasis (reported in 29 (56\%) studies after excluding studies focused on patients with Kartagener syndrome) ranged from $9 \%$ to $100 \%$, with a weighted mean of $56 \%$ (online supplementary figure S4). The heterogeneity $\left(\mathrm{I}^{2}\right)$ in prevalence of lower respiratory symptoms ranged from $89 \%$ in sputum production to $94 \%$ in cough.

\section{Upper respiratory symptoms}

Rhinitis, rhinorrhea or nasal congestion were assessed in 28 (54\%) studies and ranged in prevalence from $9 \%$ to $100 \%$ (weighted mean $75 \%$; online supplementary figure S5). Otitis media (with or without effusion) was reported in $26(50 \%)$ studies and its prevalence varied from $23 \%$ to $100 \%$ (weighted mean $74 \%$; online supplementary figure S6). Sinusitis was reported in 29 studies (56\%, after excluding studies focusing on Kartagener syndrome) with a weighted mean of $69 \%$ (range 10-100\%; online supplementary figure S7). Hearing impairment was reported in 14 (27\%) studies and prevalence ranged from $8 \%$ to $100 \%$ (weighted mean 36; figure 3). Insertion of grommets was reported in 12 (23\%) studies and prevalence ranged from $5 \%$ to $92 \%$ (weighted mean 55\%; online supplementary figure S8). Nasal polyps were described in $14(27 \%)$ studies with a weighted mean of $19 \%$ (range 3-60\%; online supplementary

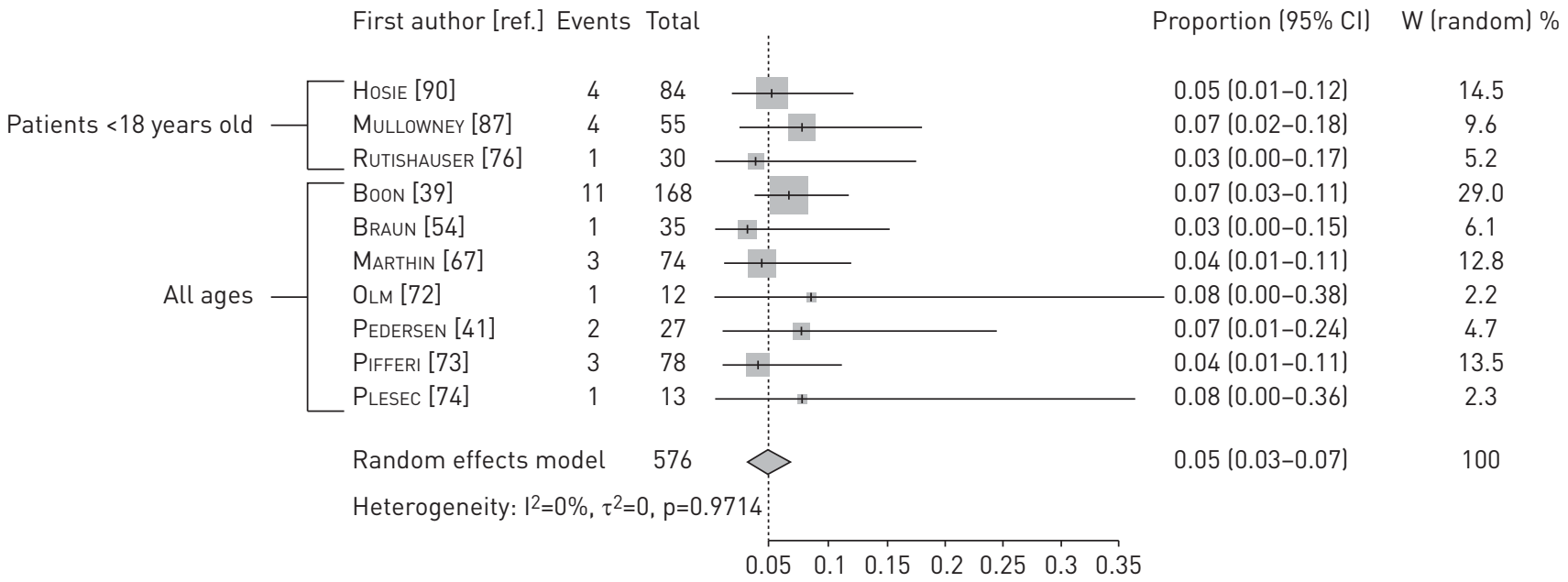

FIGURE 4 Congenital heart disease in primary ciliary dyskinesia patients: forest plot showing the heterogeneity and weighted mean prevalence in the included publications. 
figure S9). The heterogeneity in prevalence of these upper respiratory symptoms and health problems ranged from $68 \%$ for nasal polyps to $93 \%$ for rhinitis and grommet insertion.

\section{Other symptoms}

Figure 4 shows the prevalence of congenital heart disease, which was the only characteristic showing no heterogeneity $\left(\mathrm{I}^{2}=0 \%\right.$ ) with a weighted mean of $5 \%$ (ranging from $3 \%$ to $8 \%$ ) and was reported in 10 studies (19\%, excluding studies focusing on patients with Kartagener syndrome). 17 (33\%) studies assessed neonatal respiratory distress and prevalence varied from $15 \%$ to $91 \%$ (weighted mean 55\%; online supplementary figure S10) Infertility was reported in seven (13.5\%) publications which had a study population of adults or adults and children, and this was assessed only in the adult patients of these studies. Prevalence ranged from $15 \%$ to $79 \%$ (weighted mean 30\%; online supplementary figure S11). Of the seven studies reporting on infertility, four stratified for sex. In these four studies, 58\% of females evaluated were infertile; male fertility was reported in three studies and $100 \%$ of males evaluated in these studies were infertile. The heterogeneity in prevalence ranged from $0 \%$ in congenital heart disease to $91 \%$ in neonatal respiratory distress. Other symptoms reported in a small number of studies were recurrent headaches, episodes of fever and gastroesophageal reflux. Other health conditions such as hydrocephalus and retinitis pigmentosa, which have been described as rare manifestations of PCD were only reported in three and two studies, respectively, and renal manifestations were not reported in any of the studies.

\section{Differences in prevalence in different age groups and severity of symptoms}

The clinical manifestations assessed were rarely described stratified by sex or age group. The 11 studies describing symptoms separately in adults and children included usually a small number of adults and they had no further stratification into smaller age groups. No information on symptom severity was reported.

\section{Subgroup meta-analyses}

After excluding seven studies where diagnosis was only based on clinical manifestations or where no information on diagnosis was available, subgroup meta-analyses performed in 45 studies with diagnosis proven using electron microscopy, or electron microscopy plus other tests showed similar results to the ones presented. Weighted mean prevalence, range and heterogeneity of all symptoms from the subgroup meta-analyses are presented in detail in online supplementary figs S12-S25.

\section{Meta-regression}

Meta-regression showed that the explanatory factors considered explained only a small part of the between-studies variance for all symptoms. Year of publication explained some of the heterogeneity for sputum production and sinusitis, with a higher prevalence in studies published before 2004 ( $\mathrm{p}=0.06$ and 0.005, respectively). Another factor that explained part of the heterogeneity was the type of clinic from which the study originated. Paediatric pulmonology clinics and ENT clinics in particular showed a higher prevalence of hearing impairment $(\mathrm{p}<0.0001)$ compared to general paediatric clinics. Age of patients included also explained part of the heterogeneity, as situs anomalies and bronchiectasis were more common in studies including adults, or adults and children, compared to the ones including only children ( $\mathrm{p}=0.004$ and 0.02 , respectively). Bronchiectasis was also more common in prospective studies $(\mathrm{p}=0.03)$. Otitis media and hearing impairment had higher prevalence in studies where diagnosis was made using electron microscopy and other tests compared to the ones where diagnosis was only based on clinical symptoms. Detailed results of the meta-regression can be found in online supplementary table S2.

\section{Discussion}

This is the first systematic review of clinical manifestations in PCD patients. We found a prevalence of 5\% of congenital heart disease and a wide range in the prevalence of all other reported clinical symptoms. This heterogeneity could not be explained by the available explanatory factors. Only $7 \%$ of the originally identified 1210 studies described clinical manifestations of the disease, and 30 reported overlapping study populations. Most studies were retrospective and small, with a mean of 38 patients per study. They often originated from specialised departments (e.g. pulmonary or ENT) and focused primarily on lower and upper respiratory symptoms. Fewer than half of the studies that included both children and adults reported their information stratified by age. No study described symptom severity. Year of publication, clinic of origin, age of included patients, study design and diagnostic certainty were associated with symptom prevalence.

The main strength of this study is the methodological approach: to identify eligible studies, the search was performed without language restrictions and we included conference abstracts. Because some abstracts did not mention clinical manifestations, we screened the full text of all articles with an original PCD study population, even if clinical information was not explicitly mentioned in the abstracts. This ensured that we included studies we would have missed if we had followed the custom search protocol. We identified and 
excluded studies where the population inclusion criteria introduced a clear bias in the prevalence of certain manifestations. Additionally, we proceeded to explain the heterogeneity in results by performing a meta-regression with all available explanatory factors, but we were not able to include other known possible factors such as personal interest of the authors. PCD diagnosis has changed over time and varies between countries and centres. For this reason, we performed a sensitivity analysis excluding all studies where the diagnosis had not been confirmed using recommended tests.

We restricted our search to studies published since 1980 for several reasons. First, older studies are often not available online or do not have an available abstract on the online databases. Second, since 1933 when PCD was first described by Kartagener, many things have changed in diagnosing, understanding and characterising the disease. Third, the studies that described clinical characteristics of PCD before 1980 were mostly case reports or small studies with $<10$ patients, which would not be eligible for our review.

The limitations of this review reflect the limitations of the included studies, namely inadequate study designs and the presence of significant selection and misclassification bias. With regards to study design, most studies were small, single-centre case series studies that collected clinical data retrospectively from patient charts. Three main sources of selection bias are apparent, as follows. 1) Diagnostic misclassification resulting from a wide variation of diagnostic criteria used in the studies, ranging from clinical diagnosis to diagnosis established using multiple available tests (electron microscopy, video microscopy, nNO and genetics). Although there is still no established diagnostic gold standard for diagnosing PCD, the recommended diagnostic algorithm has changed considerably over the years. To address this issue we performed subgroup meta-analyses excluding the studies where the diagnosis was only clinical or not described and we tested the available diagnostic information as a possible explanatory factor of the heterogeneity in our meta-regression. 2) Since most studies originated from specialised clinics, it is expected that patients with more severe manifestations were included and these study populations cannot be considered representative of all PCD patients. 3) Many studies had restrictive inclusion criteria, including, for example, only patients with situs anomalies or with reported otitis, which would increase the prevalence of manifestations related to the selection criteria (i.e. situs status and hearing impairment). Thus we excluded studies where the population inclusion criteria introduced a clear bias in the prevalence of certain manifestations (e.g. studies including only patients with Kartagener syndrome for the prevalence of situs anomalies, sinusitis and bronchiectasis). Misclassification bias is introduced when inconsistent criteria and different definitions are used to detect and define clinical manifestations. Most studies focused on symptoms from the upper and lower respiratory system. Other symptoms were rarely reported and hardly any study reported symptoms separately for different age groups. Therefore, it was not possible to describe changes in the clinical picture throughout the life course. Information on symptom severity, such as frequency of cough or volume of sputum, was not reported. Data were collected at different points of disease; some at the time of diagnosis, others at a later follow-up appointment. As most studies suffered from the same design flaws, we did not apply any quality assessment criteria to decide which studies to include in our meta-analysis.

Due to the considerable heterogeneity, the calculated mean weighted prevalence of described clinical manifestations characteristics (with the exception of congenital heart disease) should be interpreted with caution. The meta-analysis was performed to quantify the variability in prevalence and not to give valid estimates on prevalence. The possible explanatory factors tested failed to explain this heterogeneity. Still, some factors contributed to explaining differences in prevalence of some symptoms. Year of publication reflects differences in diagnosis, but also increasing awareness of PCD. Older studies included more patients with severe disease. Age is one of the most important factors in health and disease. Situs anomalies and bronchiectasis were more common in adult patients, probably because cases without situs anomalies or severe lung disease were underdiagnosed in adults, especially in the past. The type of clinic from which the study population originated can influence reported symptoms. The discovery of bronchiectasis could be highly influenced by study design; a standardised protocol for chest computed tomography (CT) imaging and the existence of two evaluators of results instead of one are among the most important factors that could explain the higher prevalence in prospective studies. Diagnostic certainty was associated with higher prevalence of upper respiratory manifestations (otitis media and hearing impairment) in studies with test-proven diagnosis. Mild hearing impairment can remain undetected unless specific tests are performed, which could be more common after test-proven diagnosis. In addition, it is possible that unspecific upper respiratory symptoms play a less important role in the differential diagnosis of PCD compared to situs anomalies and lower respiratory symptoms. The only outcome where the meta-analysis did not suggest heterogeneity $\left(\mathrm{I}^{2}=0 \%\right)$ was congenital heart disease, where we found a prevalence of $5 \%$. This is perhaps not surprising, since severe heart disease is diagnosed early in childhood in most cases. Hence it is likely that severe congenital heart defects are least susceptible to measurement bias. The manifestations of bronchiectasis, hearing impairment and infertility would all be expected to have increased in frequency with time, with the uniform application of sensitive testing for 
detection (e.g. chest CT scanning, audiograms and spermatozoa analysis, respectively). The variable "neonatal respiratory distress" is probably increasingly subject to recall bias as patients age. We were unable to detect this in our meta-regression analysis (online supplementary table S2). However, in one study [2], where uniform methods were applied to include cases prospectively, the prevalence of neonatal respiratory distress was much higher in children (87\%) compared to adults (65\%).

In our study, methodological variability between included studies could not explain the heterogeneity in prevalence of manifestations. We believe that heterogeneity in prevalence is caused by the large variety of inclusion criteria and the insufficient standardisation of outcomes, which cannot be tested in a meta-analysis. Another possible explanation is that patients with PCD might have several distinctive phenotypes, similar to patients with cystic fibrosis [92, 93] and childhood asthma [94, 95]; the proportion of different phenotypes might vary between centres.

Our review highlights the difficulty in describing the full clinical picture of PCD based on published studies. Future studies should conform to the following criteria: 1) report on all clinical manifestations, including the less common ones; 2) assess indicators of symptoms severity; 3) use clear, homogeneous definitions of all clinical manifestations; 4) use clear inclusion criteria for the study population; 5) collect data prospectively at specified assessment time points starting from diagnosis and continuing throughout life; and 6) stratify the analysis by the degree of diagnostic certainty of PCD of the patients.

These criteria could be fulfilled by performing prospective well-designed multicentre studies in patients with carefully assessed PCD diagnosis. Another important resource will be the international PCD registry which has been established in the framework of the European Union-funded Bestcilia project [96].

This carefully performed systematic review and meta-analysis of clinical manifestations of PCD found considerable heterogeneity between studies, not explained by methodological variations. Further prospective studies with larger and carefully selected populations and well defined outcomes will allow better characterisation of the disease, and possibly define different phenotypes of PCD.

\section{Acknowledgements}

We would like to thank Janne Estill, Ekaterina Safroneeva and Jingying Wang (Institute of Social and Preventive Medicine, University of Bern, Bern, Switzerland) for their help in translating published studies from Finnish, Russian and Chinese.

\section{References}

1 Barbato A, Frischer T, Kuehni CE, et al. Primary ciliary dyskinesia: a consensus statement on diagnostic and treatment approaches in children. Eur Respir J 2009; 34: 1264-1276.

2 Noone PG, Leigh MW, Sannuti A, et al. Patrimary ciliary dyskinesia: diagnostic and phenotypic features. Am J Respir Crit Care Med 2004; 169: 459-467.

3 Lucas JS, Walker WT, Kuehni CE, et al. Primary ciliary dyskinesia. In: Courdier J-F, ed. Orphan Lung Diseases. Eur Respir Monogr 2011; 54: 201-217.

4 Turner JA, Corkey CW, Lee JY, et al. Clinical expressions of immotile cilia syndrome. Pediatrics 1981; 67: 805-810.

5 Boon M, Jorissen M, Proesmans M, et al. Primary ciliary dyskinesia, an orphan disease. Eur J Pediatr 2013; 172 : $151-162$.

6 Kuehni CE, Frischer T, Strippoli MP, et al. Factors influencing age at diagnosis of primary ciliary dyskinesia in European children. Eur Respir J 2010; 36: 1248-1258.

7 Strippoli MP, Frischer T, Barbato A, et al. Management of primary ciliary dyskinesia in European children: recommendations and clinical practice. Eur Respir J 2012; 39: 1482-1491.

$8 \quad$ Miller JJ. The inverse of the Freeman-Tukey double arcsine transformation. Am Stat 1978; 32: 138.

9 Freeman MF, Tukey JW. Transformations related to the angular and the square root. Ann Math Stat 1950; 607-611.

10 Higgins J, Thompson SG, Spiegelhalter DJ. A re-evaluation of random-effects meta-analysis. J R Stat Soc Ser A Stat Soc 2009; 172: 137-159.

11 Higgins JP, Thompson SG. Quantifying heterogeneity in a meta-analysis. Stat Med 2002; 21: 1539-1558.

12 Chao J, Turner JA, Sturgess JM. Genetic heterogeneity of dynein-deficiency in cilia from patients with respiratory disease. Am Rev Respir Dis 1982; 126: 302-305.

13 Levison H, Mindorff CM, Chao J, et al. Pathophysiology of the ciliary motility syndromes. Eur J Respir Dis Suppl 1983; 127: 102-117.

14 Nadel HR, Stringer DA, Levison H. The immotile cilia syndrome: radiological manifestations. Radiology 1985; 154: 651-655.

15 Mygind N, Pedersen M, Nielsen MH. Primary and secondary ciliary dyskinesia. Acta Otolaryngol 1983; 95: 688-694.

16 Pedersen M, Mygind N. Rhinitis, sinusitis and otitis media in Kartagener's syndrome (primary ciliary dyskinesia). Clin Otolaryngol Allied Sci 1982; 7: 373-380.

17 Armengot M, Juan G, Barona R, et al. Immotile cilia syndrome: nasal mucociliary function and nasal ciliary abnormalities. Rhinology 1994; 32: 109-111.

18 Carda C, Armengot M, Escribano A, et al. Ultrastructural patterns of primary ciliar dyskinesia syndrome. Ultrastruct Pathol 2005; 29: 3-8.

19 Armengot Carceller M, Carda Batalla C, Escribano A, et al. Estudio del transporte mucociliar y de la ultraestructura ciliar nasales en pacientes con sindrome de Kartagener. [Study of mucociliary transport and nasal ciliary ultrastructure in patients with Kartagener's syndrome]. Arch Bronconeumol 2005; 41: 11-15. 

primary ciliary dyskinesia syndrome. A frequent pathology]. Arch Bronconeumol 1994; 30: 19-22.

21 Pifferi M, Bush A, Michelucci A, et al. Mannose-binding lectin 2 gene polymorphism and lung damage in primary ciliary dyskinesia. Pediatr Pulmonol 2015; 50: 179-186.

22 Santamaria F, Esposito M, Montella S, et al. Sleep disordered breathing and airway disease in primary ciliary dyskinesia. Respirology 2014; 19: 570-575.

23 Mirra V, Caffarelli C, Maglione M, et al. Hypovitaminosis D: a novel finding in primary ciliary dyskinesia. Ital J Pediatr 2015; 41: 14

24 Gokdemir Y, Karadag-Saygi E, Erdem E, et al. Comparison of conventional pulmonary rehabilitation and high-frequency chest wall oscillation in primary ciliary dyskinesia. Pediatr Pulmonol 2014; 49: 611-616.

25 Rollin M, Seymour K, Hariri M, et al. Rhinosinusitis, symptomatology \& absence of polyposis in children with primary ciliary dyskinesia. Rhinology 2009; 47: 75-78.

26 Coren ME, Meeks M, Morrison I, et al. Primary ciliary dyskinesia: age at diagnosis and symptom history. Acta Paediatr 2002; 91: 667-669.

27 Munro NC, Currie DC, Lindsay KS, et al. Fertility in men with primary ciliary dyskinesia presenting with respiratory infection. Thorax 1994; 49: 684-687.

28 Greenstone M, Stanley P, Cole P, et al. Upper airway manifestations of primary ciliary dyskinesia. J Laryngol Otol 1985; 99: 985-991.

29 Engesaeth VG, Warner JO, Bush A. New associations of primary ciliary dyskinesia syndrome. Pediatr Pulmonol 1993; 16: 9-12.

30 Prulière-Escabasse V, Coste A, Chauvin P, et al. Otologic features in children with primary ciliary dyskinesia. Arch Otolaryngol Head Neck Surg 2010; 136: 1121-1126.

31 Blanchon S, Legendre M, Copin B, et al. Delineation of CCDC39/CCDC40 mutation spectrum and associated phenotypes in primary ciliary dyskinesia. J Med Genet 2012; 49: 410-416.

32 Tamalet A, Clement A, Roudot-Thoraval F, et al. Abnormal central complex is a marker of severity in the presence of partial ciliary defect. Pediatrics 2001; 108: E86.

33 Pittman JE, Rosenfeld M, LaFave C, et al. Characteristics of primary ciliary dyskinesia in children under 5 years of age. Am J Respir Crit Care Med 2011; 183: A1213.

34 Ferkol TW, Puffenberger EG, Lie H, et al. Primary ciliary dyskinesia-causing mutations in Amish and Mennonite communities. J Pediatr 2013; 163: 383-387.

35 Shapiro AJ, Davis SD, Olivier KN, et al. Clinical symptoms associated with primary ciliary dyskinesia: results of a multi-centered study. Am J Respir Crit Care Med 2010; 181: A6728.

36 Shapiro AJ, Tolleson-Rinehart S, Zariwala MA, et al. The prevalence of clinical features associated with primary ciliary dyskinesia in a heterotaxy population: results of a web-based survey. Cardiol Young 2015; 25: 752-759.

37 Knowles MR, Ostrowski LE, Leigh MW, et al. Mutations in RSPH1 cause primary ciliary dyskinesia with a unique clinical and ciliary phenotype. Am J Respir Crit Care Med 2014; 189: 707-717.

38 Kim RH, A Hall D, Cutz E, et al. The role of molecular genetic analysis in the diagnosis of primary ciliary dyskinesia. Ann Am Thorac Soc 2014; 11: 351-359.

39 Boon M, De Boeck K, Jorissen M, et al. Primary ciliary dyskinesia and humoral immunodeficiency - is there a missing link? Respir Med 2014; 108: 931-934.

40 van der Baan S, Veerman AJ, Weltevreden EF, et al. Kartagener's syndrome: clinical symptoms and laboratory studies. Eur J Respir Dis Suppl 1983; 127: 91-95.

41 Pedersen M, Stafanger G. Bronchopulmonary symptoms in primary ciliary dyskinesia. A clinical study of 27 patients. Eur I Respir Dis Suppl 1983; 127: 118-128.

42 Mygind N, Pedersen M. Nose-, sinus- and ear-symptoms in 27 patients with primary ciliary dyskinesia. Eur $J$ Respir Dis Suppl 1983; 127: 96-101.

43 McManus IC, Mitchison HM, Chung EM, et al. Primary ciliary dyskinesia (Siewert's/Kartagener's syndrome): respiratory symptoms and psycho-social impact. BMC Pulm Med 2003; 3: 4.

44 Jain K, Padley SP, Goldstraw EJ, et al. Primary ciliary dyskinesia in the paediatric population: range and severity of radiological findings in a cohort of patients receiving tertiary care. Clin Radiol 2007; 62: 986-993.

45 Shapiro AJ, Davis SD, Ferkol T, et al. Laterality defects other than situs inversus totalis in primary ciliary dyskinesia: insights into situs ambiguus and heterotaxy. Chest 2014; 146: 1176-1186.

46 Davis SD, Ferkol TW, Rosenfeld M, et al. Clinical features of childhood primary ciliary dyskinesia by genotype and ultrastructural phenotype. Am J Respir Crit Care Med 2015; 191: 316-324.

47 Afzelius BA, Eliasson R. Male and female infertility problems in the immotile-cilia syndrome. Eur J Respir Dis Suppl 1983; 127: 144-147.

48 Alsaadi MM, Habib SS, Al Muqhem BA, et al. Significance of fractional exhaled nitric oxide measurements in detecting primary ciliary dyskinesia in Saudi children. Saudi Med J 2013; 34: 24-28.

49 Armengot M, Bonet M, Carda C, et al. Development and validation of a method of cilia motility analysis for the early diagnosis of primary ciliary dyskinesia. Acta Otorrinolaringol Esp 2012; 63: 1-8.

50 Armengot M, Juan G, CardaC, et al. Prevalencia del sindrome de discinesia ciliar primaria en pacientes con sinusitis y bronquiectasias. [The prevalence of primary dyskinetic ciliary syndromes in patients with sinusitis and bronchiectasis]. An Otorrinolaringol Ibero Am 1995; 22: 85-92.

51 Bai Y, Zhang J, You S, et al. Primaire ciliaire dyskinesie; een enquete-onderzoek naar het klinische beeld. [Clinical characteristics of primary ciliary dyskinesia]. Zhonghua Er Bi Yan Hou Tou Jing Wai Ke Za Zhi 2014; 49: $115-120$.

52 Barlocco EG, Valletta EA, Canciani M, et al. Ultrastructural ciliary defects in children with recurrent infections of the lower respiratory tract. Pediatr Pulmonol 1991; 10: 11-17.

53 Beucher J, Chambellan A, Segalen J, et al. Dyskinésie ciliaire primitive: revue rétrospective clinique et paraclinique. [Primary ciliary dyskinesia: a retrospective review of clinical and paraclinical data]. Rev Mal Respir 2011; 28: 856-863.

54 Braun JJ, Donato L, Clavert A, et al. La dyskinésie ciliaire primitive. [Primary ciliary dyskinesia. Clinical presentation and diagnosis]. Ann Otolaryngol Chir Cervicofac 2005; 122: 63-68.

55 Busquets RM, Caballero-Rabasco MA, Velasco M, et al. Primary ciliary dyskinesia: clinical criteria indicating ultrastructural studies. Arch Bronconeumol 2013; 49: 99-104. 
, 157: 422-426.

2000; 159: 857-860.

63 Iñiguez CR, Fonseca AX, Hernández CJ, et al. Disquinesia ciliar: diagnóstico ultraestructural, evolución clínica y alternativas de tratamiento. [Clinical and ultrastructural features of ciliary dyskinesia]. Rev Med Chil 2007; 135: 1147-1152.

64 Kawakami M, Hattori Y, Nakamura S. Reflection of structural abnormality in the axoneme of respiratory cilia in the clinical features of immotile cilia syndrome. Intern Med 1996; 35: 617-623.

65 Korppi M, Dunder T, Remes S, et al. Värekarvojen synnynnäiset toimintahäiriöt lapsilla. [Congenital ciliary dysfunction in children]. Duodecim 2011; 127: 2294-2302.

66 Lesic I, Maurer E, Strippoli MP, et al. Primäre Ziliendyskinesie in Österreich. [Primary ciliary dyskinesia (PCD) in Austria]. Wien Klin Wochenschr 2009; 121: 616-622.

67 Marthin JK, Petersen N, Skovgaard LT, et al. Lung function in patients with primary ciliary dyskinesia: a cross-sectional and 3-decade longitudinal study. Am J Respir Crit Care Med 2010; 181: 1262-1268.

68 Min YG, Shin JS, Choi SH, et al. Primary ciliary dyskinesia: ultrastructural defects and clinical features. Rhinology 1995; 33: 189-193.

69 Montella S, Santamaria F, Salvatore M, et al. Lung disease assessment in primary ciliary dyskinesia: a comparison between chest high-field magnetic resonance imaging and high-resolution computed tomography findings. Ital $J$ Pediatr 2009; 35: 24.

70 O'Callaghan C, Chetcuti P, Moya E. High prevalence of primary ciliary dyskinesia in a British Asian population. Arch Dis Child 2010; 95: 51-52.

71 Oktem S, Karadag B, Erdem E, et al. Sleep disordered breathing in patients with primary ciliary dyskinesia. Pediatr Pulmonol 2013; 48: 897-903.

72 Olm MA, Kögler JE Jr, Macchione M, et al. Primary ciliary dyskinesia: evaluation using cilia beat frequency assessment via spectral analysis of digital microscopy images. J Appl Physiol (1985) 2011; 111: 295-302.

73 Pifferi M, Bush A, Di Cicco M, et al. Health-related quality of life and unmet needs in patients with primary ciliary dyskinesia. Eur Respir J 2010; 35: 787-794.

74 Plesec TP, Ruiz A, McMahon JT, et al. Ultrastructural abnormalities of respiratory cilia: a 25-year experience. Arch Pathol Lab Med 2008; 132: 1786-1791.

75 Rachinskir SV, Volkov IK, Sereda EV, et al. [The Zivert-Kartagener syndrome in children]. Probl Tuberk 1993: $19-22$.

76 Rutishauser M. Klinik der primären ziliären Dyskinesie. [Clinical features of primary ciliary dyskinesia]. Schweiz Med Wochenschr 2000; 130: 705-710.

77 Smit HJ, Schreurs AJ, Van den Bosch JM, et al. Is resection of bronchiectasis beneficial in patients with primary ciliary dyskinesia? Chest 1996; 109: 1541-1544.

78 Sommer JU, Schäfer K, Omran H, et al. ENT manifestations in patients with primary ciliary dyskinesia: prevalence and significance of otorhinolaryngologic co-morbidities. Eur Arch Otorhinolaryngol 2011; 268: 383-388.

79 Sturgess JM, Thompson MW, Czegledy-Nagy E, et al. Genetic aspects of immotile cilia syndrome. Am J Med Genet 1986; 25: 149-160.

80 Tolusakow WL, Boikow GA, Lewaschow JN, et al. Zeitschrift für Erkrankungen der Atmungsorgane. [Bronchiectasis in patients with situs inversus viscerum (Kartagener syndrome) (author's transl)]. Z Erkr Atmungsorgane 1981; 156: 167-175.

81 Vallet C, Escudier E, Roudot-Thoraval F, et al. Primary ciliary dyskinesia presentation in 60 children according to ciliary ultrastructure. Eur J Pediatr 2013; 172: 1053-1060.

82 van der Baan S. Primary ciliary dyskinesia and the middle ear. Laryngoscope 1991; 101: 751-754.

83 Wang LF, Guan YB, Gu YY, et al. Bronchiolitis in Kartagener syndrome: imaging diagnosis and following up. Chin J Med Imag Technol 2009; 25: 2040-2042.

84 Wolter NE, Dell SD, James AL, et al. Middle ear ventilation in children with primary ciliary dyskinesia. Int $J$ Pediatr Otorhinolaryngol 2012; 76: 1565-1568.

$85 \mathrm{Xu} \mathrm{BP}$, Shen KL, Hu YH, et al. [Clinical characteristics of primary ciliary dyskinesia in children]. Zhonghua Er Ke Za Zhi 2008; 46: 618-622.

86 Yiallouros PK, Kouis P, Middleton N, et al. Clinical features of primary ciliary dyskinesia in Cyprus with emphasis on lobectomized patients. Respir Med 2015; 109: 347-356.

87 Mullowney T, Manson D, Kim R, et al. Primary ciliary dyskinesia and neonatal respiratory distress. Pediatrics 2014; 134: 1160-1166.

88 Boon M, Smits A, Cuppens H, et al. Primary ciliary dyskinesia: critical evaluation of clinical symptoms and diagnosis in patients with normal and abnormal ultrastructure. Orphanet J Rare Dis 2014; 9: 11-20.

89 Chin GY, Karas DE, Kashgarian M. Correlation of presentation and pathologic condition in primary ciliary dyskinesia. Arch Otolaryngol Head Neck Surg 2002; 128: 1292-1294.

90 Hosie P, Fitzgerald DA, Jaffe A, et al. Primary ciliary dyskinesia: overlooked and undertreated in children. J Paediatr Child Health 2014; 50: 952-958.

91 Enderby BJ, Desai M, Heaver R. A cross-sectional analysis of children treated for primary ciliary dyskinesia at Birmingham Children's Hospital. Am J Respir Crit Care Med 2010; 181: A3320. 
92 Durno C, Corey M, Zielenski J, et al. Genotype and phenotype correlations in patients with cystic fibrosis and pancreatitis. Gastroenterology 2002; 123: 1857-1864.

93 Hubert D, Bienvenu T, Desmazes-Dufeu N, et al. Genotype-phenotype relationships in a cohort of adult cystic fibrosis patients. Eur Respir J 1996; 9: 2207-2214.

94 Spycher B, Silverman M, Kuehni C. Phenotypes of childhood asthma: are they real? Clin Exp Allergy 2010; 40: $1130-1141$.

95 Spycher BD, Henderson J, Granell R, et al. Genome-wide prediction of childhood asthma and related phenotypes in a longitudinal birth cohort. J Allergy Clin Immunol 2012; 130: 503-509.

96 Werner C, Lablans M, Ataian M, et al. An international registry for primary ciliary dyskinesia. Eur Respir J 2016; 47: 849-859. 